University of Nebraska - Lincoln

DigitalCommons@University of Nebraska - Lincoln

February 2006

\title{
A Bidomain Nonribosomal Peptide Synthetase Encoded by FUM14 Catalyzes the Formation of Tricarballylic Esters in the Biosynthesis of Fumonisins
}

\author{
Kathia Zaleta-Rivera \\ Chunping $\mathrm{Xu}$ \\ Fengan $\mathrm{Yu}$ \\ Robert A. E. Butchko \\ Robert H. Proctor \\ See next page for additional authors \\ Follow this and additional works at: https://digitalcommons.unl.edu/chemistrydussault \\ Part of the Chemistry Commons
}

Zaleta-Rivera, Kathia; Xu, Chunping; Yu, Fengan; Butchko, Robert A. E.; Proctor, Robert H.; Hidalgo-Lara, Maria E.; Raza, Ashraf S.; Dussault, Patrick; and Du, Liangcheng, "A Bidomain Nonribosomal Peptide Synthetase Encoded by FUM14 Catalyzes the Formation of Tricarballylic Esters in the Biosynthesis of Fumonisins" (2006). Patrick Dussault Publications. 7.

https://digitalcommons.unl.edu/chemistrydussault/7

This Article is brought to you for free and open access by the Published Research - Department of Chemistry at DigitalCommons@University of Nebraska - Lincoln. It has been accepted for inclusion in Patrick Dussault Publications by an authorized administrator of DigitalCommons@University of Nebraska - Lincoln. 


\section{Authors}

Kathia Zaleta-Rivera, Chunping Xu, Fengan Yu, Robert A. E. Butchko, Robert H. Proctor, Maria E. HidalgoLara, Ashraf S. Raza, Patrick Dussault, and Liangcheng Du 


\title{
A Bidomain Nonribosomal Peptide Synthetase Encoded by FUM14 Catalyzes the Formation of Tricarballylic Esters in the Biosynthesis of Fumonisins ${ }^{\dagger}$
}

\author{
Kathia Zaleta-Rivera, ${ }^{\ddagger}$, Chunping Xu, ${ }^{\ddagger}$ Fengan Yu, Robert A. E. Butchko," Robert H. Proctor," \\ María E. Hidalgo-Lara, ${ }^{\S}$ Ashraf Raza, ${ }^{\perp}$ Patrick H. Dussault, ${ }^{\ddagger}$ and Liangcheng Du, ${ }^{*, \neq}$ \\ Departments of Chemistry and Biochemistry, University of Nebraska-Lincoln, Lincoln, Nebraska 68588, National Center for \\ Agriculture Utilization Research, ARS-USDA, 1815 North University Street, Peoria, Illinois 61604, and Departamento de \\ Biotecnología y Bioingeniería, CINVESTAV-IPN, Col. Zacatenco, CP 07360, Mexico D.F., Mexico
}

Received October 12, 2005; Revised Manuscript Received December 17, 2005

\begin{abstract}
Fumonisins are a group of polyketide-derived mycotoxins produced by Fusarium verticillioides, a filamentous fungus infecting corn and contaminating food and feeds. Fumonisins contain two tricarballylic esters that are critical for toxicity. Here, we present genetic and biochemical data for the esterification mechanism. FUM14 in F. verticillioides has been deleted by homologous recombination, and the resultant mutant lost the ability to produce fumonisins. Two new metabolites, $\mathrm{HFB}_{3}$ and $\mathrm{HFB}_{4}$, which are biosynthetic precursors of fumonisins lacking the tricarballylic esters, were detected in the mutant. The results suggest that FUM14 is required for the esterification of fumonisins. FUM14 was predicted to encode a nonribosomal peptide synthetase (NRPS) containing two domains, peptidyl carrier protein and condensation domain. Both the intact Fum14p and the condensation domain have been expressed in Escherichia coli and purified for activity assays. Fum14p was able to convert $\mathrm{HFB}_{3}$ and $\mathrm{HFB}_{4}$ to the tricarballylic esters-containing fumonisins, $\mathrm{FB}_{3}$ and $\mathrm{FB}_{4}$, respectively, when incubated with tricarballylic thioester of $N$-acetylcysteamine. In addition, the condensation domain was able to convert $\mathrm{HFB}_{1}$ to $\mathrm{FB}_{1}$. These data provide direct evidence for the role of Fum $14 \mathrm{p}$ in the esterification of fumonisins. More interestingly, the results are the first example of an NRPS condensation domain catalyzing a $\mathrm{C}-\mathrm{O}$ bond (ester) formation, instead of the typical $\mathrm{C}-\mathrm{N}$ bond (amide) formation in nonribosomal peptides. The understanding of the esterification mechanism provides useful knowledge for mycotoxin reduction and elimination. The study also provides new insight into the reactions catalyzed by NRPS.
\end{abstract}

Fumonisins are polyketide-derived mycotoxins produced by several filamentous fungi, including Fusarium verticillioides, which is a widespread pathogen of corn $(1,2)$. These mycotoxins are structurally similar to the long-chain base of sphingolipids and thus can disrupt the sphingolipid pathway by inhibiting sphinganine $N$-acyltransferase (ceramide synthase) (3). This disruption is thought to be responsible for several fatal animal diseases, such as leukoencephalomalacia in horses and pulmonary edema in pigs, and the epidemiological association of fumonisins and esophageal cancer and neural tube defects in humans $(1,4,5)$. The fungus is associated with disease at all stages of maize plant development, infecting the roots, stalk, and kernels. Wildtype strains of the fungus predominantly produce the $\mathrm{B}$-series fumonisins, including fumonisin $\mathrm{B}_{1}\left(\mathrm{FB}_{1}\right),{ }^{1}$ fumonisin $\mathrm{B}_{2}$ $\left(\mathrm{FB}_{2}\right)$, fumonisin $\mathrm{B}_{3}\left(\mathrm{FB}_{3}\right)$, and fumonisin $\mathrm{B}_{4}\left(\mathrm{FB}_{4}\right)$ (Figure 1).

This work was supported in part by a Layman Award from UNL, an Elsa Pardee Foundation Award, and NSFC (no. 30428023). K.Z.$\mathrm{R}$. is supported by a CONACYT Scholarship (no. 166245), Mexico.

* To whom correspondence should be addressed at Department of Chemistry, University of Nebraska, 729 Hamilton Hall, Lincoln, NE 68588. Phone, 1-402-472-2998; fax, 1-402-472-9402; e-mail, 1du@unlserve.unl.edu.

Department of Chemistry, University of Nebraska-Lincoln.

$\S$ CINVESTAV-IPN.

"ARS-USDA.

${ }^{\perp}$ Department of Biochemistry, University of Nebraska-Lincoln.

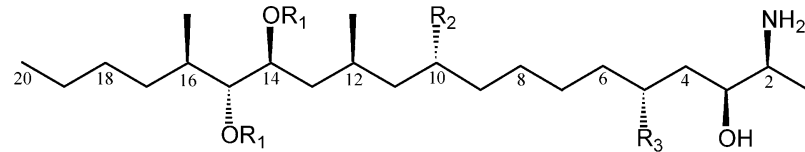<smiles>CC(C)(C)CC(=O)CC(CC(=O)O)C(=O)O</smiles>

FIGURE 1: Chemical structure of the B-series fumonisins.

The chemical structure of fumonisins is characterized by having two tricarballylic esters on the carbon backbone (Figure 1) $(2,6)$. The esterification is an essential step in the maturation of fumonisins, because without the esters the mycotoxins are not fully active (7-9). The esters are formed between a tricarboxylic acid (propane-1,2,3-tricarboxylic acid) and the hydroxyls at $\mathrm{C}-14$ and $\mathrm{C}-15$ of the fumonisin backbone (Figure 1). Previous studies using ${ }^{13} \mathrm{C}$-enriched

${ }^{1}$ Abbreviations: C, condensation; CoA, coenzyme A; EDTA, ethylenediaminetetraacetic acid; ELSD, evaporative light-scattering detection; FB, Fumonisin B series; HPLC, high-performance liquid chromatography; IPTG, $\beta$-isopropyl-thiogalactoside; ESIMS, electrospray ionization mass spectrometry; MBP, maltose binding protein; NRPS, nonribosomal peptide synthetase; PCP, peptidyl carrier protein; PCR, polymerase chain reaction; SDS-PAGE, sodium dodecyl sulfatepolyacrylamide gel electrophoresis; SNAC, thioester of $N$-acetylcysteamine; TCA, tricarballylic acid. 
substrates suggested that the precursor for the tricarballylic esters may be an intermediate from the Krebs Cycle (10). However, the biosynthetic mechanism for the ester formation is not clear.

Proctor et al. identified a 15-gene cluster (FUM) responsible for the biosynthesis of fumonisins in $F$. verticillioides (11). On the basis of the sequence annotation and preliminary results from gene deletion experiments, Butchko et al. have proposed that three genes, FUM7, FUM10, and FUM14, are involved in the formation of the esters (12). The deduced amino acid sequence of FUM1O is similar to acyl-CoA synthetase and the adenylation domain of nonribosomal peptide synthetase (NRPS); the deduced amino acid sequence of FUM14 is similar to the peptidyl carrier protein (PCP) and condensation domain (C) of NRPS (13), and the deduced amino acid sequence of $F U M 7$ is similar to dehydrogenases, which could be regarded as a reductase domain of NRPS $(14,15)$. Thus, FUM7, FUM10, and FUM14 may encode enzymes that make up an NRPS complex.

In NRPS, the adenylation domain activates an amino acid and transfers it to PCP as a covalently linked thioester via a $4^{\prime}$-phosphopantetheinyl cofactor $(13,16)$. The $\mathrm{C}$ domain then catalyzes the condensation reaction between the acyl thioesters on PCP to form an amide $(\mathrm{C}-\mathrm{N})$ bond. Therefore, among the four genes, FUM14 is the most likely candidate that is directly involved in the transfer of the acyl groups to the hydroxyls on polyketide backbone. The FUM14 protein (Fum14p) could catalyze formation of the ester $\mathrm{C}-\mathrm{O}$ bonds in the biosynthesis of fumonisins. A $\mathrm{C}-\mathrm{O}$ bond formation catalyzed by an NRPS condensation domain has not been reported in the biosynthesis of nonribosomal peptides. Therefore, the characterization of FUMI4 not only helps reveal the biosynthetic mechanism for the side-chain formation of fumonisins but also could shed new light on the reactions catalyzed by NRPS. Here, we report the gene disruption and heterologous expression of FUM14 and in vitro activity assays for the purified Fum14p.

\section{EXPERIMENTAL PROCEDURES}

General DNA Manipulations. Plasmids preparation and DNA extraction were carried out by using commercial kits (Qiagen, Valencia, CA), and all other manipulations were carried out according to standard methods (17). Escherichia coli DH5 $\alpha$ strain was used as the host for general DNA propagations, and the pGEM-zf vector series from Promega (Madison, WI) was used for cloning and DNA sequencing. Genomic DNA of $F$. verticillioides was prepared as described previously (18).

Construction of FUM14 Deletion Vector pUCH2-F14. To construct the FUM14 vector, two DNA fragments were amplified by PCR from Cos4-5 (11), which contains most of the FUM gene cluster including FUM13, FUM14, and FUM15 (see Figure 2). A 991-bp fragment (left-side arm), which covers the noncoding region downstream to FUM14 and the 3 '-region of $F U M 13$, was amplified by using primers PLF/PLR (Table 1). A 1087-bp fragment (right-side arm), which covers the $5^{\prime}$-region of FUM14 and the noncoding region upstream to $F U M 14$, was amplified by using primers PRF/PRR (Table 1). The right-side arm was digested with HindIII and BamHI and cloned into plasmid pUCH2-8, which carries a hygromycin B resistance gene $(\mathrm{HygB})(19)$.
The left-side arm was digested with ApaI/SalI to generate an 834-bp fragment, which was subsequently cloned into the ApaI/SalI site of the above construct. This generated a 7.3kb plasmid, pUCH2-F14, in which the $H y g B$ gene is flanked by the left-side arm and right-side arm as shown in Figure 2. The plasmid was used to create FUM14-deleted mutants by homologous recombination at the left-side and right-side arms.

Transformation of $F$. verticillioides and Screening for Mutants. The procedure for $F$. verticillioides protoplast isolation and transformation was essentially identical to that described previously (20). Briefly, the plasmid DNA (5 $\mu \mathrm{g})$ was diluted with STC buffer ( $100 \mu \mathrm{L}$ final) and mixed with protoplasts $(100 \mu \mathrm{L})$, and transformation was mediated with PEG 8000 buffer (30\% PEG 8000, 10 mM Tris-HCl, pH 8.0 , and $50 \mathrm{mM} \mathrm{CaCl}_{2}$ ). Hygromycin-resistant colonies were selected on YPD plates containing hygromycin B $(150 \mu \mathrm{g} /$ $\mathrm{mL}$, Calbiochem, La Jolla, CA). Three different pairs of primers were used in PCR to identify the FUM14-deleted mutants from the hygromycin resistant colonies. First, a pair of primers binding to $\operatorname{HygB}$ was used to verify that the colonies contained this gene. Then, two pairs of primers (P1/ $\mathrm{P} 2$ and $\mathrm{P} 3 / \mathrm{P} 4$, see Table 1 for sequences) were used to verify that colonies positive for $\mathrm{Hyg} B$ resulted from homologous recombination at the flanking regions of FUM14, but not from a random integration of the plasmid. Primer P1 binds to a region that is outside of the left-side arm, whereas primer P2 binds to the $H y g B$ gene (Figure 2). Therefore, only the colonies resulting from homologous recombination at the leftside arm could yield a PCR product of the expected size, whereas colonies containing a randomly integrated vector or resulting from rearrangement after the homologous recombination would not yield this specific product. Similarly, primers $\mathrm{P} 3 / \mathrm{P} 4$ were used to confirm the homologous recombination at the right-side arm (Figure 2). Thus, FUM14-deleted mutants were identified from colonies that yielded the PCR product expected for homologous recombination on both the left-side and right-side arms.

Production and Extraction of Metabolites from Mutants. The mutant strains were transferred separately to a YPD/ hygromycin $(300 \mu \mathrm{g} / \mathrm{mL})$ plate to generate single colonies. A single colony was then transferred to a $10 \mathrm{~mL}$ tube containing $3 \mathrm{~mL}$ of YPD/hygromycin $(150 \mu \mathrm{g} / \mathrm{mL})$ liquid medium and allowed to grow in a shaker (60 rpm) at room temperature for 2 days. From the $3 \mathrm{~mL}$ culture, $100 \mu \mathrm{L}$ was transferred to a flask containing $25 \mathrm{~mL}$ of YPD/hygromycin $(150 \mu \mathrm{g} / \mathrm{mL})$. The culture was incubated with shaking at room temperature for another 2 days. The culture was transferred to a $50 \mathrm{~mL}$ tube and centrifuged at $2500 \mathrm{rpm}$ for $20 \mathrm{~min}$. The pellet was washed three times with sterile water and finally resuspended in $10 \mathrm{~mL}$ of sterile water. From the suspended solution, $500 \mu \mathrm{L}$ was transferred to a new flask containing $10 \mathrm{~g}$ of autoclaved CMK (Cracked Maize Kernels) medium (11). Alternatively, colonies derived from single spores of the mutants were inoculated on V8 agar plates. After 1-week growth at room temperature, spores were collected from the plates and used to inoculate the $10 \mathrm{~g} \mathrm{CMK}$ medium. After 3 weeks of incubation at room temperature in the dark, metabolites were extracted from the CMK cultures with $20 \mathrm{~mL}$ of $50 \%$ acetonitrile. The extracts were filtered, and a sample of $50 \mu \mathrm{L}$ was injected in HPLC-ELSD to analyze the metabolites. 

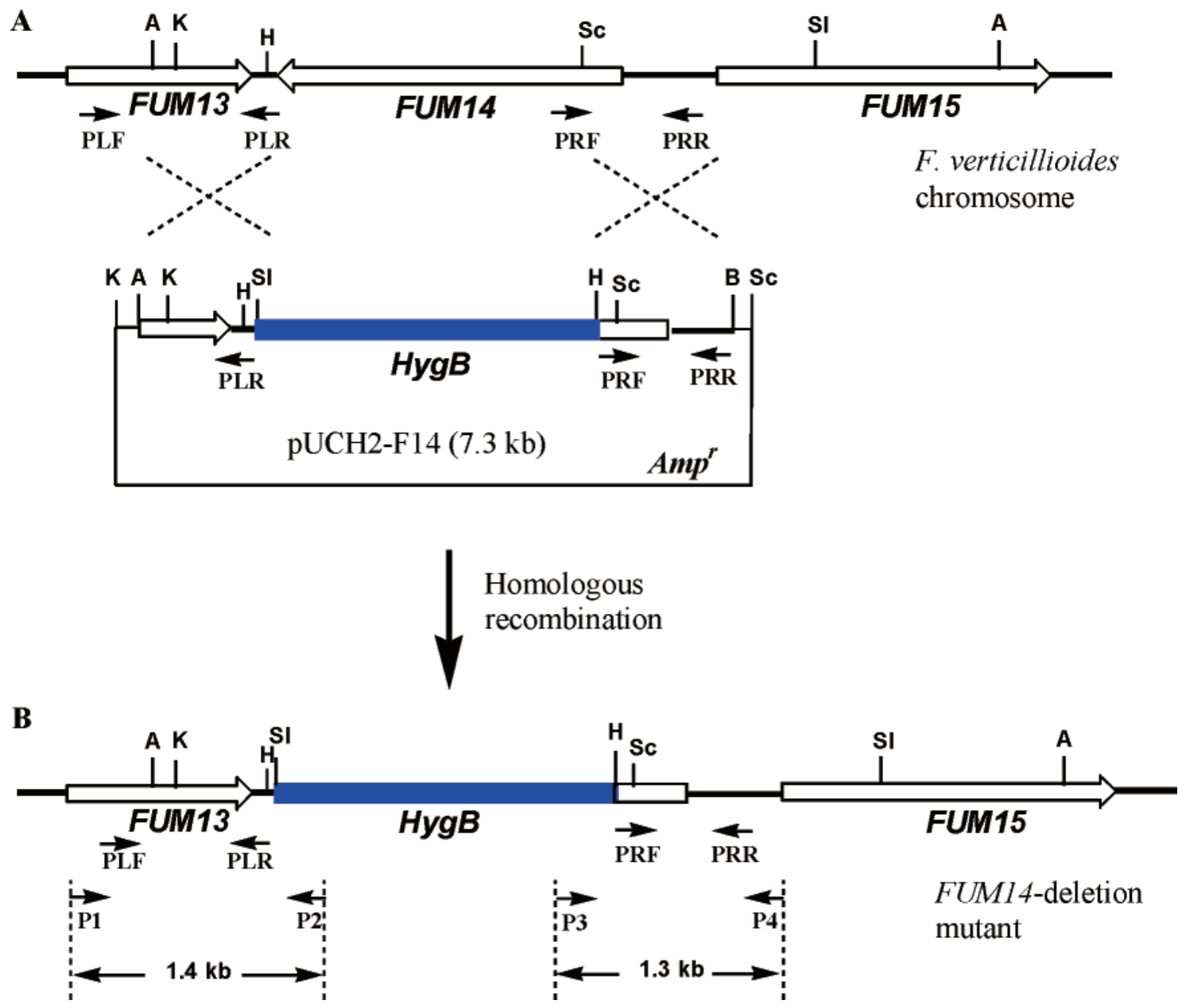

FIGURE 2: The construction of FUM14 gene deletion vector and screening of the FUM14-deleted mutants. (A) Homologous recombination between FUM14 on the F. verticillioides chromosome and the homologous sequences on plasmid pUCH2-F14. The positions for PCR primer binding sites are indicated by small arrows. Abbreviations: A, ApaI; B, BamHI; H, HindIII; K, KpnI; Sc, SacI; Sl, SalI; HygB, hygromycin B resistant gene. (B) Gene-replacement mutants resulted from double crossovers at the upstream homologous region (left-side arm) and at the downstream homologous region (right-side arm). The expected size for PCR products is indicated below the corresponding genes.

\begin{tabular}{ll}
\hline $\begin{array}{c}\text { Table 1: } \\
\text { name }\end{array}$ & \multicolumn{1}{c}{ Primers Used in the Experiments } \\
\hline PLF & 5'-CCG CTC GAG TAT TAC AAG GGC GAC TC-3' \\
PLR & 5'-CGC GTC GAC GAT AGC TAG AGC ATA TTC GG-3' \\
PRF & 5' CGT GAA GCT TGG GAA CTC GTC CTT TCT-3' \\
PRR & 5'CGC GGA TCC GAA GCA CAG AGT CGG AAA ACG-3' \\
P1 & 5'-GTA CAA GCA CGG ACT TGA AAG AG-3' \\
P2 & 5'-GAT GTG TTA GAA GCT CAC AGA AGG-3' \\
P3 & 5'-CAT AAC CAA GCC TAT GCC TAC AG-3' \\
P4 & 5'-GCA TAC TGC CTG TTC TAG GAT TG-3' \\
F14-C-F & 5'-CGC GGA TCC TTG CCT TTA TGG TCT ATG-3' \\
F14-C-R & 5'-CCC AAG CTT CTA GTC CAA CAA GCC TGT-3' \\
F14-F & 5'-CGC GGA TCC AAT TCA TTG GAC CAG TGG-3' \\
F14-R & 5'-CCG CTC GAG CTA GTC CAA CAA GCC TGT-3' \\
\hline
\end{tabular}

Construction of Expression Vectors. To construct the expression vector that contained the $\mathrm{C}$ domain alone (Fum14C), a $1.3 \mathrm{~kb}$ fragment of FUM14 carrying the domain was amplified by PCR from the genomic DNA of $F$. verticillioides with primers F14-C-F and F14-C-R (Table 1). Highfidelity DNA polymerase $P f u$ (Stratagene) was used in the reactions. The PCR fragment was digested with BamHI and HindIII and cloned into pGEM-3zf. After sequence fidelity of the amplification product was confirmed by DNA sequencing, it was transferred to expression vector pMALc2 (New England BioLabs), which is designed to yield the expressed protein fused to the maltose-binding protein (MBP).

To construct the complete Fum $14 \mathrm{p}$ expression vector, a $1.6 \mathrm{~kb}$ fragment was amplified by PCR from a cDNA library of $F$. verticillioides using the forward primer F14-F and a reverse primer F14-R (Table 1). The PCR fragment was digested with BamHI and XhoI and cloned into pT7Blue-3. After confirmation by DNA sequencing, the gene was transferred to expression vector pMAL-c2 as described above.

Expression and Purification of Fum14-C and Fum14-PCPC. E. coli TB1 (New England BioLabs) strain was used for expression from the pMAL-based constructs. The standard conditions for cell growth and expression induction were followed as recommended by the manufacturer. The cells were incubated in a shaker $(250 \mathrm{rpm})$ at $25^{\circ} \mathrm{C}$ until the cell density reached 0.6 at $\mathrm{OD}_{600 \mathrm{~nm}}$. To induce the expression of FUM14, IPTG $(0.1 \mathrm{mM})$ was added to the culture, and the cells were allowed to grow at the same conditions for another $3 \mathrm{~h}$. The soluble fraction of the overproduced proteins was loaded to an amylose resin column (BioLabs) for affinity purification of the proteins. The purified proteins were desalted on a PD-10 column (Pharmacia Biotech, Piscataway, $\mathrm{NJ}$ ), eluted in $50 \mathrm{mM}$ Tris- $\mathrm{HCl}$ buffer, $\mathrm{pH} 8.0$, containing 

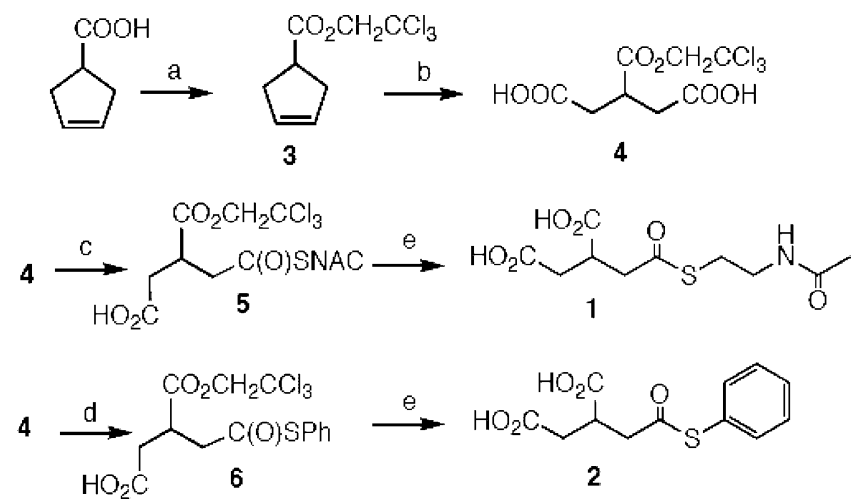

FIGURE 3: Synthesis of TCA thioesters. (a) $\mathrm{CCl}_{3} \mathrm{CH}_{2} \mathrm{OH}, \mathrm{DCC}$, pyridine; (b) $\mathrm{RuCl}_{3}, \mathrm{NaIO}_{4}$; (c) $\mathrm{N}$-acetylcysteamine, DCC, pyridine; (d) thiophenol, DCC, pyridine; (e) Zn/HOAc.

$200 \mathrm{mM} \mathrm{NaCl}, 10 \mathrm{mM} \mathrm{MgCl} 2,2 \mathrm{mM}$ dithiothreitol (DTT), $1 \mathrm{mM}$ EDTA, and $10 \%$ glycerol, and stored at $-80{ }^{\circ} \mathrm{C}$ for in vitro assays.

Preparation of $\mathrm{HFB}_{1}, \mathrm{HFB}_{3}$, and $\mathrm{HFB}_{4}$. The preparation of the hydrolyzed $\mathrm{FB}_{1}\left(\mathrm{HFB}_{1}\right)$ and hydrolyzed $\mathrm{FB}_{3}\left(\mathrm{HFB}_{3}\right)$ was conducted by using the alkaline method (21). Approximately $2 \mathrm{mg}$ of fumonisins was refluxed in $2.5 \mathrm{~mL}$ of $0.25 \mathrm{~N}$ potassium hydroxide for $24 \mathrm{~h}$. The solution was acidified to $\mathrm{pH} 5$ with $2 \mathrm{~N} \mathrm{HCl}$ and analyzed by HPLCELSD. The hydrolyzed fumonisin was purified by HPLC and dried under vacuum. Alternatively, $\mathrm{HFB}_{3}$ and $\mathrm{HFB}_{4}$ were prepared from the culture of FUM14-deleted mutants. The purified HFB was dissolved in water for enzyme activity assays.

Synthesis of Tricarballylic Acid Monothioesters. N-Acetylcysteamine (1) and phenyl (2) monothioesters of tricarballylic acid were prepared as illustrated in Figure 3 (experimental details are available in Supporting Information). Protection of cyclopentene acetic acid as the corresponding trichloroethyl ester was followed by oxidative cleavage of the alkene with ruthenium tetroxide. Thioesterification of the resulting diacid with $N$-acetylcysteamine and thiophenol cleanly produced the corresponding phenyl and SNAC monothioesters. Reductive deprotection of the trichloroethyl esters furnished the tricarballylic monothioesters. As the phenyl thioester decomposed rapidly following dissolution in neutral buffers, the biosynthetic studies were conducted with the $\mathrm{N}$-acetylcysteamine thioester, which was indefinitely stable.

Activity Assays for Fum14p. A typical assay solution (100 $\mu \mathrm{L}$ ) contained $2 \mathrm{mM}$ tricarballylic thioester, $0.1 \mathrm{mM} \mathrm{HFB}$ $\left(\mathrm{HFB}_{1}, \mathrm{HFB}_{3}\right.$, or $\left.\mathrm{HFB}_{4}\right), 2.3 \mu \mathrm{M}$ Fum14p, and $5 \mathrm{mM} \mathrm{MgCl}_{2}$, in $100 \mathrm{mM}$ Tris- $\mathrm{HCl}$ buffer, $\mathrm{pH}$ 7.4. Reactions without Fum $14 \mathrm{p}$ were used as controls. After $30 \mathrm{~min}$ incubation at $37^{\circ} \mathrm{C}$, the reactions were stopped by the addition of $0.2 \mathrm{~mL}$ of cold $100 \%$ ethanol. After $30 \mathrm{~min}$ on ice, the precipitated protein was removed by centrifugation at $13200 \mathrm{rpm}$ at 4 ${ }^{\circ} \mathrm{C}$ for $20 \mathrm{~min}$, and the supernatant was transferred to a new tube and dried under vacuum. Finally, the residues in the tube were redissolved in $50 \mu \mathrm{L}$ of water for HPLC-ELSD and LC-ESIMS analysis.

HPLC-ELSD and ESIMS Analysis. The HPLC system was a ProStar, model 210 from Varian (Walnut Creek, CA) with an Alltima C18LL column $(250 \mathrm{~mm} \times 4.6 \mathrm{~mm}$ i.d., $5 \mu \mathrm{m}$, Alltech, Deerfield, IL). The mobile phases were (A) water-TFA (100:0.025, v/v) and (B) acetonitrile-TFA (100:
$0.025, \mathrm{v} / \mathrm{v})$ with a gradient of $0-40 \% \mathrm{~B}$ in $\mathrm{A}$ in the first 10 $\min , 40-80 \% \mathrm{~B}$ in A from 10 to $15 \mathrm{~min}, 80 \% \mathrm{~B}$ in $\mathrm{A}$ from 15 to $20 \mathrm{~min}, 80-100 \% \mathrm{~B}$ in $\mathrm{A}$ from 20 to $21 \mathrm{~min}, 100 \%$ $\mathrm{B}$ in A from 21 to $23 \mathrm{~min}$, and $100-0 \% \mathrm{~B}$ in A from 23 to $25 \mathrm{~min}$. The flow rate was $1.0 \mathrm{~mL} / \mathrm{min}$, and a $50 \mu \mathrm{L}$ sample was injected. The conditions set for light-scattering detection using an ELSD2000 (Alltech, Deerfield, IL) were the same as described previously (22). For preparative HPLC, the respective fractions were collected directly from the column according to their retention times. ESIMS was carried out on an API Qtrap 4000. The samples were directly infused in the mass spectrometer and analyzed in positive ion mode using Turbo Ion Spray Source.

\section{RESULTS}

FUM14-Deleted Mutants Produced $\mathrm{HFB}_{3}$ and $\mathrm{HFB}_{4}$. The transformation of $F$. verticillioides protoplasts with $\mathrm{pUCH} 2-$ F14 resulted in 33 colonies that were able to grow on YPD plates containing hygromycin $\mathrm{B}$. The resistance phenotype of these transformants was confirmed by the growth of the individual colonies in liquid YPD medium containing a high concentration $(300 \mu \mathrm{g} / \mathrm{mL})$ of hygromycin $\mathrm{B}$. The genomic DNA isolated from the 33 transformants was then used in PCR screening for FUM14-deleted mutants. When primers P1/P2 were used, PCR of 12 transformants yielded a $1.4 \mathrm{~kb}$ fragments, which is expected to result from a homologous recombination of the left-side arm sequence in pUCH2-F14 and the $F$. verticillioides genome (Figure 2B). The 12 colonies were further screened by PCR using primers P3/ P4. Except for one transformant, all transformants clearly yielded a $1.3 \mathrm{~kb}$ fragment, which is expected to result from a homologous recombination at the right-side arm sequence in pUCH2-F14 and the $F$. verticillioides genome (Figure 2B). To further verify the identity of the transformants, the PCR products from four representative transformants (number 23, 27, 31, and 33) were digested with $K p n I$ and EcoRI. Two fragments, 693 and $796 \mathrm{bp}$, were produced when the PCR product amplified by primer $\mathrm{P} 1 / \mathrm{P} 2$ was digested with $\mathrm{KpnI}$, whereas three fragments, 754, 446, and $141 \mathrm{bp}$, were generated when the PCR product amplified by primers P3/ P4 was digested with EcoRI (data not shown). The presence of $H y g B$ in each of the transformants was further verified by PCR amplification of the gene from the genomic DNA of the transformants, with the wild-type strain as a negative control (data not shown). Together, the results showed that we have obtained at least four fungal strains that are FUM14deleted mutants, in which the coding region of FUMI4 was replaced with $H y g B$ (Figure 2B). The four mutants were used in the subsequent experiments for the production and analysis of metabolites.

After 3 weeks of growth in CMK medium, the wild-type progenitor strain produced B-series fumonisins, $\mathrm{FB}_{1}, \mathrm{FB}_{2}$, $\mathrm{FB}_{3}$, and $\mathrm{FB}_{4}$, with retention times of 16.2 , 17.0, 17.2, and $18.1 \mathrm{~min}$, respectively, on HPLC-ELSD (Figure 4A). The extracts from FUM14-deleted mutants gave three main peaks on HPLC-ELSD, with retention times of 16.1, 16.9, and 18.1 (Figure 4B). Since the retention times are very close to those from the wild-type strain, the extract from the wildtype strain was combined with the extract from the mutant and re-injected to HPLC. Two groups of peaks, with each approximately corresponding to that of the wild-type strain or of the mutant, were produced on HPLC-ELSD (data not 


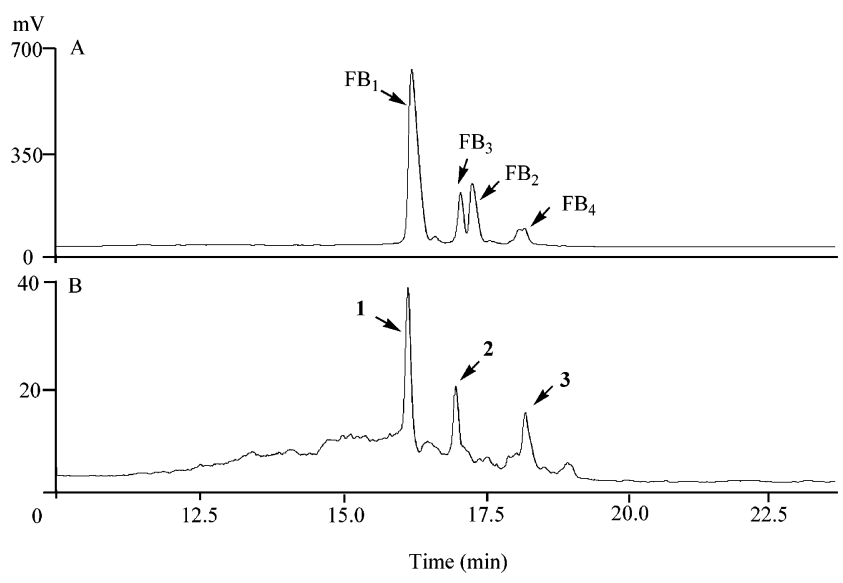

FIGURE 4: HPLC-ELSD analysis of metabolites produced in $F$. verticillioide. (A) wild-type; (B) FUM14 deletion mutant. The identity of the peaks on the wild-type panel is indicated.

shown). The results suggest that the peaks in the extract from mutants correspond to compounds that differ from fumonisins produced by the wild-type progenitor strain. In the similar spiking experiments, Peak-1 of the mutant extract did not

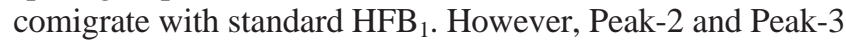
comigrated with standard $\mathrm{HFB}_{3}$ and $\mathrm{HFB}_{4}$ (data not shown). The three peaks were collected individually and analyzed by ESIMS. Peak-1 exhibited a yellowish color and gave several molecular ions, among which were $[\mathrm{M}+\mathrm{H}]^{+}$of $321.3 \mathrm{~m} / \mathrm{z}$ for methylfusarubin and 303.3 and $337.4 \mathrm{~m} / \mathrm{z}$ for the dehydrolyzed form and hydroxylated form of methylfusarubin, respectively (23). These pigments have been observed in other FUM-deleted mutants and are not related to fumonisins (Yu, F. and Du, L., unpublished observations). Peak-2 gave only one clear molecular ion of $390.6 \mathrm{~m} / \mathrm{z}$, which is identical to standard $\mathrm{HFB}_{3}$. Peak-3 contained a predominant molecular ion of $374.6 \mathrm{~m} / \mathrm{z}$, which is the same as standard $\mathrm{HFB}_{4}$. The results from HPLC and ESIMS show that FUM14-deleted mutants produce two hydrolyzed fumonisins, $\mathrm{HFB}_{3}$ and $\mathrm{HFB}_{4}$, but not fumonisins $\mathrm{HFB}_{1}$ or $\mathrm{HFB}_{2}$.

Production of Fum14p-C and Fum14p in E. coli. FUM14 was initially predicted to encode only for a condensation domain of NRPS (11). Results from the recent EST sequencing project of $F$. verticillioides showed that the start codon of FUM14 is located upstream of the previously predicted start codon (24). We found that the amino acid sequence deduced from this new coding region is similar to two domains within NRPS, a PCP domain at the N-terminus and a condensation domain at the $\mathrm{C}$-terminus. The highly conserved 4'-phosphopantetheinyl binding motif, FFDLGGDSVKA (consensus sequence GGxS; serine residue being the $4^{\prime}$-phosphopantetheinyl binding site), is found in the PCP domain of Fum $14 p$, whereas the conserved motifs, including the C3 motif DHTHCDAFSR (typical consensus sequence HHxxxDG), are found in the C domain of Fum14p (Figure 5) $(13,16)$.

We constructed expression vectors for Fum14p C domain alone (Fum14p-C) as well as for the entire protein (Fum14p). The corresponding regions of FUM14 were initially cloned into expression vector pET28a under control of a T7/lac promoter. The proteins were overproduced in $E$. coli strain BL21(DE3) upon IPTG induction. However, the produced proteins were totally insoluble under all conditions tested.
The Fum14p- and Fum14p-C-coding regions were then moved to the yeast expression system, pYES/NT, in the INVSc1 strain (Invitrogen). However, the expression level of both proteins was so extremely low that neither was purified from this system. Finally, we moved the Fum14pand Fum14p-C-coding regions to the TB2/pMALc2 system to test whether the proteins could be expressed as soluble proteins when fused to MBP. Although the expression level was lower than in the BL21(DE3)/pET28a, the produced proteins appeared partially soluble. The proteins were purified on an amylose affinity column. The single domain protein, produced from the Fum $14 \mathrm{p}-\mathrm{C}$ coding region, yielded a band at approximately the $90 \mathrm{kDa}$ region on SDS-PAGE (Figure 5A), which is consistent with the expected size for the fusion protein $(92.5 \mathrm{kDa})$. The entire Fum $14 \mathrm{p}$ protein, produced from the intact Fum $14 \mathrm{p}$ coding region, yielded a band at approximately the $100 \mathrm{kDa}$ region on SDS-PAGE (Figure 5B), which is in agreement with the expected size for the fusion protein $(104.8 \mathrm{kDa})$.

Esterification of $\mathrm{HFB}_{3}$ and $\mathrm{HFB}_{4}$ by Fum14p. Fum14p was initially tested for the ability to transfer commercially available acyl-CoA, including acetyl-CoA, malonyl-CoA, and succinyl-CoA, to hydrolyzed fumonisins. However, none of the acyl-CoA appeared to serve as substrate for the enzyme. Subsequently, we chemically synthesized the SNAC monothioester of tricarballylic acid as substrate mimic. Since $\mathrm{HFB}_{3}$ was the major metabolite accumulated in the FUM14 mutant, we first tested the activity of Fum14p using $\mathrm{HFB}_{3}$ as the acceptor substrate and TCA-SNAC as the acyl donor. HPLC analysis of the reaction mixture showed the presence of a major peak at 16.93 min (Peak-1 in Figure 6C), which comigrated with standard $\mathrm{HFB}_{3}$, and a smaller peak at 16.99 min (Peak-2 in Figure 6C), which comigrated with standard $\mathrm{FB}_{3}$. Peak-2 was not observed in the control where Fum14p was omitted in the reaction (Figure 6B). To confirm the formation of $\mathrm{FB}_{3}$ in the reaction, the reaction mixture was subjected to ESIMS analysis. A clear molecular ion of $706.7 \mathrm{~m} / \mathrm{z}$, which was identical to standard $\mathrm{FB}_{3}$, was detected, in addition to the $390.6 \mathrm{~m} / \mathrm{z}$ ion for standard $\mathrm{HFB}_{3}$. Only the $390.6 \mathrm{~m} / \mathrm{z}$ ion, but not the $706.7 \mathrm{~m} / \mathrm{z}$ ion, was present in the corresponding fraction collected from the control reaction. We also tested the activity of Fum $14 \mathrm{p}$ using $\mathrm{HFB}_{4}$ as the acceptor and TCA-SNAC as the donor. HPLC analysis of the reaction mixture showed a single peak at $18.10 \mathrm{~min}$, which comigrated with standard $\mathrm{HFB}_{4}$ (data not shown). Because $\mathrm{FB}_{4}$ and $\mathrm{HFB}_{4}$ have the same retention time under the experimental conditions, we analyzed the reaction mixture by ESIMS. The results showed that a $690.8 \mathrm{~m} / \mathrm{z}$ ion, which was identical to standard $\mathrm{FB}_{4}$, was present in the fraction from the reaction, but not from the control in which Fum $14 \mathrm{p}$ was not included. The control contained only the $374.6 \mathrm{~m} / \mathrm{z}$ ion for $\mathrm{HFB}_{4}$. The results show that Fum14p is able to esterify $\mathrm{HFB}_{3}$ and $\mathrm{HFB}_{4}$ using TCA-SNAC as the acyl donor to produce the corresponding fumonisins. Finally, we tested the activity of the $\mathrm{C}$ domain (Fum14p-C) using $\mathrm{HFB}_{3}$ or $\mathrm{HFB}_{4}$ as the acceptor. The results from HPLC and ESIMS analysis suggest that no fumonisin was formed in the reactions (data not shown).

Esterification of $\mathrm{HFB}_{1}$ by Fum $14 p$ and Fum 14p-C. To obtain information about the specificity of Fum14p toward the acceptor substrate, we further tested the esterification of 
A

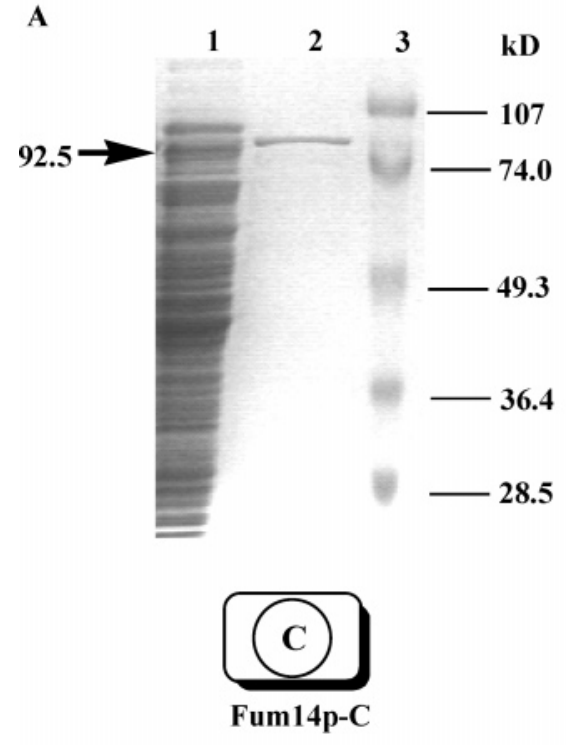

C3 motif of C-domain

\begin{tabular}{lrll}
\hline HTS1 & 1479 & NHLVFDGMST & 1488 \\
HMWP2 & 245 & DLLIMDASSF & 254 \\
EntF & 136 & HHLLVDGFSF & 145 \\
Fum14p & 244 & DHTHCDAFSR & 253
\end{tabular}

B

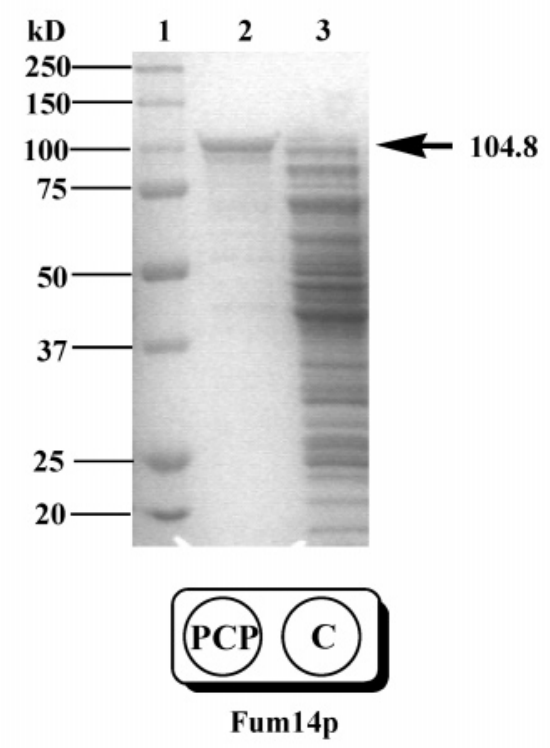

\begin{tabular}{lrll} 
PPT-binding & site of PCP-domain \\
\hline HTS1 & 796 & FIHAGGDSITA & 806 \\
HMWP2 & 45 & LIQAGLDSIRL & 55 \\
EntF & 999 & FFALGGHSLLA & 1009 \\
Fum14p & 28 & FFDLGGDSVKA & 38
\end{tabular}

FIGURE 5: Sodium dodecyl sulfate-polyacrylamide gel analysis of Fum14p expressed in E. coli. (A) The condensation domain of Fum14p. lane 1, total soluble proteins; lane 2, purified protein; lane 3, protein size markers. (B) Intact Fum14p. Lane 1, protein size markers; lane 2, purified protein; lane 3, total soluble proteins. The conserved motif for the condensation domain and peptidyl carrier protein of nonribosomal peptides is listed under the SDS-PAGE. HTS1, HC-toxin synthetase (Q01886) from Cochliobolus carbonum; HMWP2, high-molecularweight protein 2 (P48633) from Yersinia enterocolitica; EntF, enterobactin synthetase component F (P11454) from E. coli.

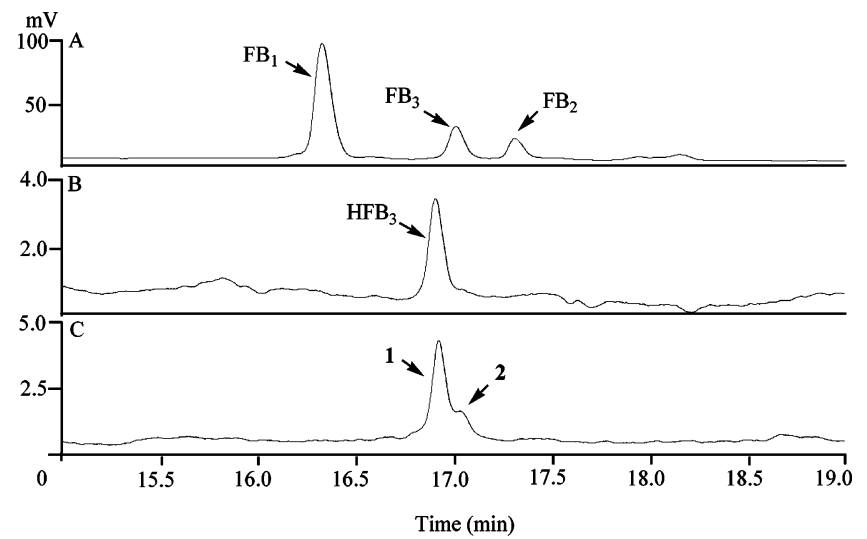

FIGURE 6: HPLC-ELSD analysis of the reaction catalyzed by Fum $14 \mathrm{p}$ using $\mathrm{HFB}_{3}$ as substrate in the presence of TCA thioester. (A) Standard $\mathrm{FB}_{1}, \mathrm{FB}_{2}$, and $\mathrm{FB}_{3}$; (B) standard $\mathrm{HFB}_{3}$; (C) reaction mixture.

$\mathrm{HFB}_{1}$, which is the hydrolyzed form of $\mathrm{FB}_{1}$ but that was not detected in extracts of FUM14 deletion mutants (see Figure 4). HFB 1 contains one more hydroxyl (C-5 hydroxyl) than $\mathrm{HFB}_{3}$ and two more hydroxyl (C-5 and C-10 hydroxyl) than $\mathrm{HFB}_{4}$. Reactions that included $\mathrm{HFB}_{1}$, the thioester TCA-SNAC, and the intact enzyme Fum14p yielded two peaks upon HPLC analysis, one peak at $15.0 \mathrm{~min}$ (Peak-1) and the other at 15.6 min (Peak-2) (Figure 7B). Peak-1 comigrated with standard $\mathrm{HFB}_{1}$ (Figure 7A), but Peak-2 did not comigrate with standard $\mathrm{FB}_{1}$, which had a retention time of 16.1 min (Figure 7C). ESIMS analysis revealed that the reaction mixture contained a new product with a $[\mathrm{M}+\mathrm{H}]^{+}$ion of $564.2 \mathrm{~m} / \mathrm{z}$, in addition to $\mathrm{HFB}_{1}$, which has a $[\mathrm{M}+\mathrm{H}]^{+}$ion of $406.5 \mathrm{~m} / \mathrm{z}$. The product with

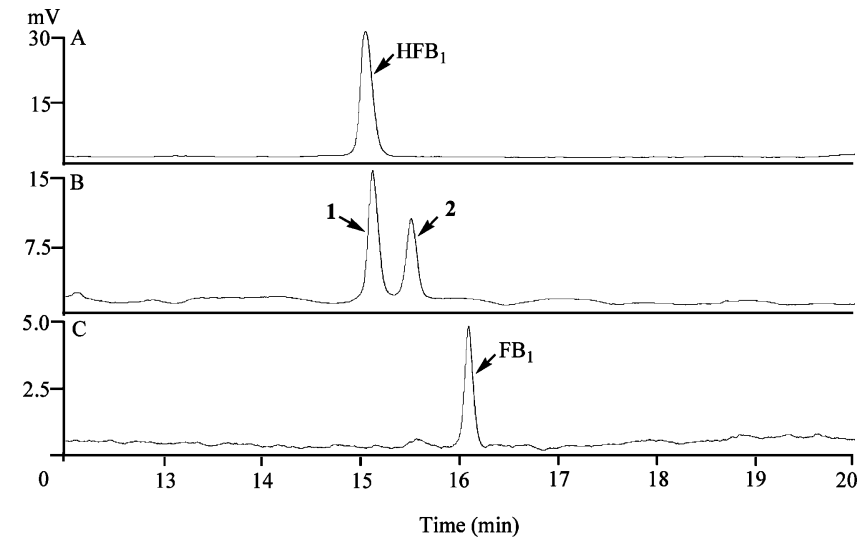

FIGURE 7: HPLC-ELSD analysis of the reaction catalyzed by Fum $14 p$ using $\mathrm{HFB}_{1}$ as substrate in the presence of TCA thioester. (A) Standard $\mathrm{HFB}_{1}$; (B) reaction mixture; (C) standard $\mathrm{FB}_{1}$.

a $[\mathrm{M}+\mathrm{H}]^{+}$ion of $564.2 \mathrm{~m} / \mathrm{z}$ is coincident with $\mathrm{PHFB}_{1}$, the partly hydrolyzed $\mathrm{FB}_{1}$ that contains only one of the two tricarballylic esters of $\mathrm{FB}_{1}(25)$. It is not clear at this time which regioisomer (C-14 ester or C-15 ester) of $\mathrm{PHFB}_{1}$ might be formed in the reaction. When the $\mathrm{C}$ domain (Fum14p-C) was used in the reaction instead of the intact protein, two peaks were also detected on HPLC (Figure 8B). The first peak (Peak-2) comigrated with $\mathrm{PHFB}_{1}$, whereas the second peak (Peak-3) comigrated with $\mathrm{FB}_{1}$ (Figure $8 \mathrm{C}$ ). The identity of the two peaks was confirmed by ESIMS. The $[\mathrm{M}+\mathrm{H}]^{+}$ for Peak-2 was $564.6 \mathrm{~m} / \mathrm{z}$, which is identical to that for $\mathrm{PHFB}_{1}$, and the $[\mathrm{M}+\mathrm{H}]^{+}$for Peak-3 was $722.7 \mathrm{~m} / \mathrm{z}$, which is identical to that for $\mathrm{FB}_{1}$. The results show that both the Fum $14 p$ and Fum $14 p-C$ proteins were able to esterify HFB in vitro. 


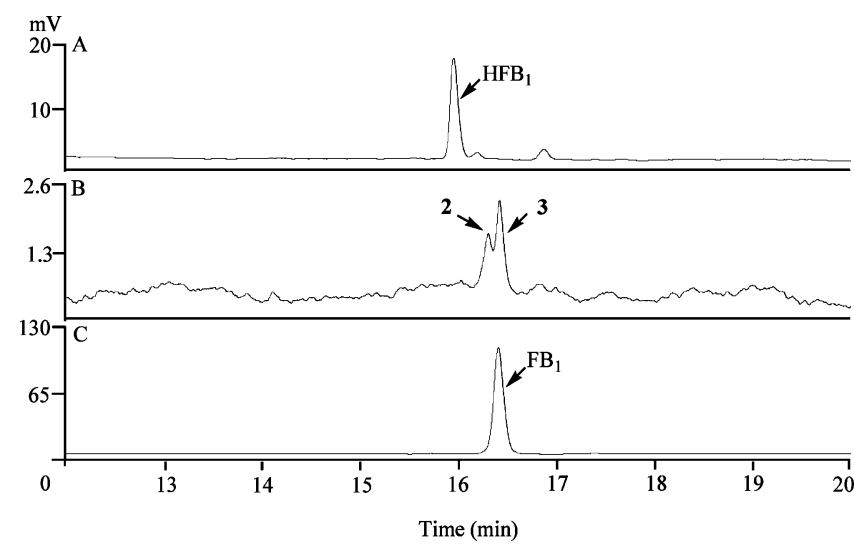

FIGURE 8: HPLC-ELSD analysis of the reaction catalyzed by the condensation domain of Fum14p using $\mathrm{HFB}_{1}$ as substrate in the presence of TCA thioester. (A) Standard $\mathrm{HFB}_{1}$; (B) reaction mixture; (C) standard $\mathrm{FB}_{1}$.

\section{DISCUSSION}

Typical NRPS are modular enzymes, with each module (except initiation module and termination module) minimally containing three domains, the adenylation domain, $\mathrm{PCP}$, and the condensation domain $(13,16)$. The enzymes are multifunctional complexes consisting of several modules in a single polypeptide. For example, the NRPS cyclosporine A synthetase includes 11 modules (26). FUM14 is predicted to encode an NRPS with only the PCP and C domains. To define the role of FUM14 in fumonisin biosynthesis, we generated FUM14 deletion mutants of $F$. verticillioides, in which the protein coding region of FUM14 was replaced with a hygromycin resistance gene. This approach was used previously to define the function of other FUM genes (20,
27, 28). HPLC and ESIMS analysis of extracts of the FUM14 deletion mutants revealed that the mutants did not produce any of the typical $\mathrm{B}$-series fumonisins $\mathrm{FB}_{1}, \mathrm{FB}_{2}, \mathrm{FB}_{3}$, and $\mathrm{FB}_{4}$. Instead, the mutants produced pre-fumonisins, $\mathrm{HFB}_{3}$ and $\mathrm{HFB}_{4}$, which are identical to $\mathrm{FB}_{3}$ and $\mathrm{FB}_{4}$, respectively, except that they lack the TCA ester functions. The fact that the mutants lost the ability to produce the TCA-esterified fumonisins indicates that FUM14 is involved in the esterification of fumonisins. The results are consistent with the preliminary observations determined previously (12). The absence of partly esterified products (PHFB) in the mutant extracts suggests that FUM14 is responsible for the TCA esters at both $\mathrm{C}-14$ and $\mathrm{C}-15$ of the fumonisin backbone.

PCP and $\mathrm{C}$ domains of NRPS have not been reported previously to catalyze the formation of the $\mathrm{C}-\mathrm{O}$ bone of ester functions. Thus, the PCP and C domains of Fum $14 p$ could be unusual in their activity. To further characterize this unusual activity, we conducted a heterologous expression analysis of Fum14p. This analysis has been challenging due to the difficulty in the heterologous expression of the genes from $F$. verticillioides $(29,30)$. We were able to express FUM14 in E. coli and purify the protein to near homogeneity only after it was fused to maltose binding protein (MBP). The in vitro assays showed that the intact enzyme was able to convert $\mathrm{HFB}_{3}$ to $\mathrm{FB}_{3}$ and $\mathrm{HFB}_{4}$ to $\mathrm{FB}_{4}$, using TCA thioester as the acyl donor. The results have two important implications. First, the biochemical data provide direct evidence for the function of Fum $14 \mathrm{p}$, that is, that it catalyzes the esterification of TCAs to both C-14 and C-15 of the fumonisin backbone. The biochemical data are also consistent with the results from the FUM14 deletion analysis. FUM14 represents the third gene among the 15-gene cluster for

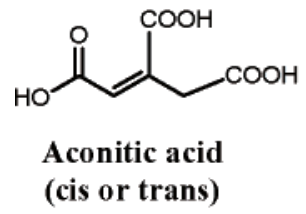
Aconitic acid
(cis or trans) Acyl-S-Fum 14p

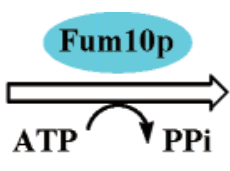

Adenylation

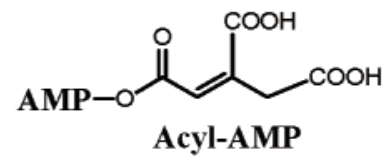

Acyl-AMP

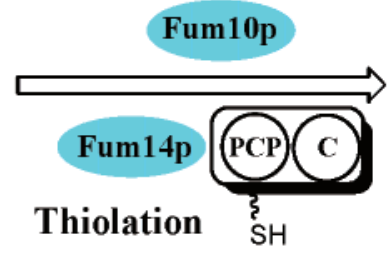

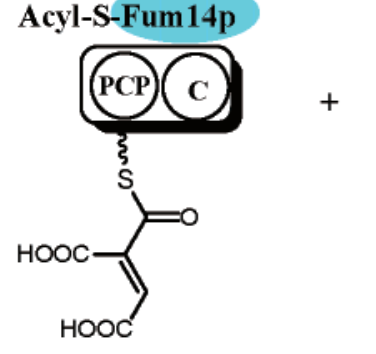
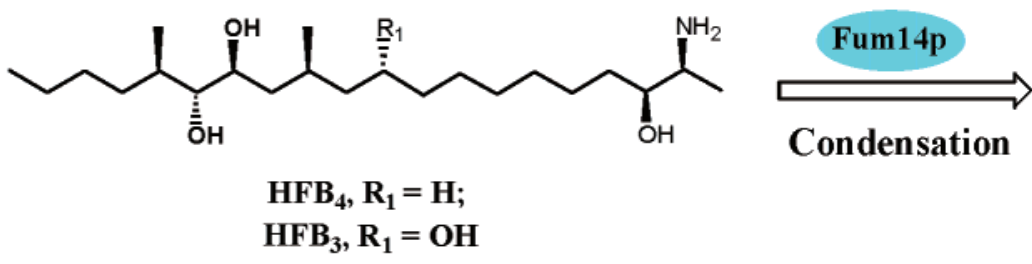

Condensation

$$
\mathrm{HFB}_{3}, \mathbf{R}_{\mathbf{1}}=\mathbf{O H}
$$
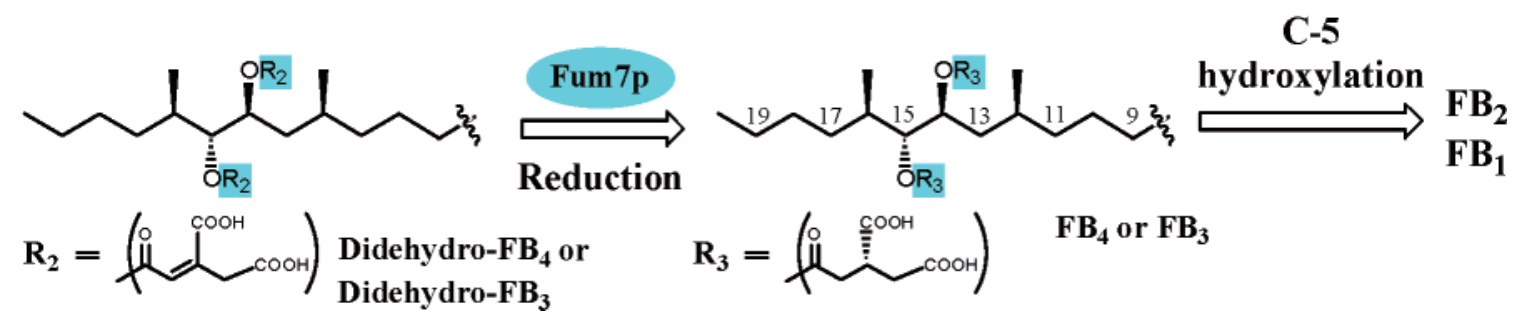

$$
\mathbf{R}_{\mathbf{3}}=\text { (并) }
$$

$\mathrm{FB}_{4}$ or $\mathrm{FB}_{3}$

FIGURE 9: A proposed mechanism for the NRPS-catalzyed tricarballylic ester formation of fumonisins. Note that an unsaturated tricarboxylate, such as aconitic acid, is proposed to be the origin of the tricarballylic esters in vivo. Three FUM genes, FUM10, FUM14, and FUM7, together code for a four-domain NRPS complex to catalyze the adenylation, thiolation, condensation, and reduction of the tricarballylic esters of fumonisins. 
biosynthesis of fumonisins that has been biochemically characterized $(29,30)$. Because of the importance of the esters in the fumonisin toxicity, it is significant to define the genetic and biochemical basis of their formation. Second, the in vitro data demonstrate the first example of NRPScatalyzed $\mathrm{C}-\mathrm{O}$ bond formation. Fum $14 p$ contains PCP and $\mathrm{C}$ domain, in which the $\mathrm{C}$ domain is the catalytic unit according to the paradigm of NRPS-catalyzed reactions (13, 16). In typical NRPS, the $C$ domain catalyzes the condensation reaction of a peptidyl-S-PCP and an aminoacyl-S-PCP to produce a new amide bond $(13,16)$. The substrates are covalently linked to the enzyme via the 4'-phosphopantetheinyl group of the PCP domain. Acyl-SNAC is a mimic of acyl-S-PCP, which is commonly used as substrate in the in vitro studies of NRPS and polyketide synthases $(31,32)$. In its natural environment, Fum14 $p$ is predicted to catalyze a condensation reaction between an enzyme-bound substrate (TCA-SNAC mimic) and a free substrate (hydrolyzed FB) to form an ester bond. It is noteworthy that the $\mathrm{C}$ domain alone (Fum14p-C) appeared inactive when $\mathrm{HFB}_{3}$ or $\mathrm{HFB}_{4}$ was used as the substrate, but active when $\mathrm{HFB}_{1}$ was the substrate. The reason for the difference is not clear but could be related to the C-5 hydroxyl group on $\mathrm{HFB}_{1}$ that may provide a favorable interaction with the enzyme. The fact that $\mathrm{HFB}_{1}$ was converted to $\mathrm{PHFB}_{1}$ and $\mathrm{FB}_{1}$ by Fum14p-C shows that the removal of the PCP domain did not lead to an inactive enzyme.

Three genes, FUM7, FUM10, and FUM14, have been suggested to be involved in the esterification of fumonisins (12). The genes together may encode an NRPS complex. FUM10 is similar to genes coding for the adenylation domains of NRPS, whereas FUM14 encodes the PCP and condensation domain of NRPS. FUM7 is similar to genes for dehydrogenases/reductases, which could be regarded as a reductase $(\mathrm{R})$ domain of NRPS. Together, the genes could encode a four-domain NRPS complex, A-PCP-C-R. On the basis of the data obtained from this study and previous studies $(11,12)$, we propose a mechanism for the biosynthesis of the TCA esters of fumonisins (Figure 9). The first step is the ATP-dependent activation of a tricarboxylate substrate by Fum10p to form an acyl-AMP, which is subsequently transferred to the PCP domain of Fum14p. The exact substrate for Fum10p is not known yet, but was suggested to be an intermediate of the Krebs Cycle (10). Preliminary data obtained from the disrupted mutants of FUMIO and FUM7 suggested that the substrate of Fum10p could be a tricarboxylate containing a double bond (12), such as aconitic acid (Figure 9). The second step is the $\mathrm{C}$ domain-catalyzed condensation between acyl-S-PCP of Fum $14 p$ and $\mathrm{HFB}_{3}$ or $\mathrm{HFB}_{4}$ to produce a didehydro-intermediate of $\mathrm{FB}_{3}$ or $\mathrm{FB}_{4}$, respectively. If this is the case, the direct substrate in vivo for the $\mathrm{C}$ domain could be an unsaturated tricarboxylate thioester, rather than the TCA thioester that we used in our enzyme assays in vitro. Attempts to chemically synthesize a monothioester of aconitic acid as an unsaturated analog of TCA-SNAC have been frustrated by the difficulties of dealing with the reactive functionality in the molecule $(\mathrm{Xu}$, C. and Dussault, P. H., unpublished results). The last step is the reduction of the double bond in the didehydro-intermediate by Fum7p to produce $\mathrm{FB}_{3}$ and $\mathrm{FB}_{4}$ (Figure 9). A hydroxylation at carbon- 5 will convert $\mathrm{FB}_{3}$ and $\mathrm{FB}_{4}$ to $\mathrm{FB}_{1}$ and $\mathrm{FB}_{2}$, respectively. This step is believed to be the last step in the whole biosynthetic pathway and has been characterized $(27,29)$. We are currently working on the characterization of Fum10p and Fum7p to provide further evidence for the proposed mechanism for esterification of fumonisins.

\section{ACKNOWLEDGMENT}

We thank Dr. R. D. Plattner at USDA, Peoria, IL, for providing standard fumonisins.

\section{SUPPORTING INFORMATION AVAILABLE}

Procedures for the synthesis of tricarballylic acid monothioesters, $N$-acetylcysteamine (1) and phenyl (2) monothioesters of tricarballylic acid. This material is available free of charge via the Internet at http://pubs.acs.org.

\section{REFERENCES}

1. Marasas, W. F. (2001) Discovery and occurrence of the fumonisins: a historical perspective, Environ. Health Perspect. 109 (Suppl. 2), 239-243.

2. Nelson, P. E., Desjardins, A. E., and Plattner, R. D. (1993) Fumonisins, mycotoxins produced by Fusarium species: biology, chemistry, and significance, Апnи. Rev. Phytopathol. 31, 233252.

3. Merrill, A. H., Jr., van Echten, G., Wang, E., and Sandhoff, K. (1993) Fumonisin B1 inhibits sphingosine (sphinganine) $\mathrm{N}$ acyltransferase and de novo sphingolipid biosynthesis in cultured neurons in situ, J. Biol. Chem. 268, 27299-27306.

4. Gelderblom, W. C., Jaskiewicz, K., Marasas, W. F., Thiel, P. G., Horak, R. M., Vleggaar, R., and Kriek, N. P. (1988) Fumonisinsnovel mycotoxins with cancer-promoting activity produced by Fusarium moniliforme, Appl. Environ. Microbiol. 54, 1806-1811.

5. Marasas, W. F., Kellerman, T. S., Gelderblom, W. C., Coetzer, J. A., Thiel, P. G., and van der Lugt, J. J. (1988) Leukoencephalomalacia in a horse induced by fumonisin B1 isolated from Fusarium moniliforme, Onderstepoort J. Vet. Res. 55, 197-203.

6. Bezuidenhout, S. C., Gelderblom, W. C. A., Gorst-Allman, C. P., Horak, R. M., Marasas, W. F. O., Spiteller, G., and Vleggaar, R. (1988) Structure elucidation of the fumonisins, mycotoxins from Fusarium moniliforme, J. Chem. Soc. Chem. Commun. 743-745.

7. Seefelder, W., Humpf, H. U., Schwerdt, G., Freudinger, R., and Gekle, M. (2003) Induction of apoptosis in cultured human proximal tubule cells by fumonisins and fumonisin metabolites, Toxicol. Appl. Pharmacol. 192, 146-153.

8. Yin, J. J., Smith, M. J., Eppley, R. M., Troy, A. L., Page, S. W., and Sphon, J. A. (1996) Effects of fumonisin B1 and (hydrolyzed) fumonisin backbone AP1 on membranes: a spin-label study, Arch. Biochem. Biophys. 335, 13-22.

9. Humpf, H. U., Schmelz, E. M., Meredith, F. I., Vesper, H., Vales, T. R., Wang, E., Menaldino, D. S., Liotta, D. C., and Merrill, A. H., Jr. (1998) Acylation of naturally occurring and synthetic 1-deoxysphinganines by ceramide synthase. Formation of $\mathrm{N}$ palmitoyl-aminopentol produces a toxic metabolite of hydrolyzed fumonisin, AP1, and a new category of ceramide synthase inhibitor, J. Biol. Chem. 273, 19060-19064.

10. Blackwell, B. A., Edwards, O. E., Fruchier, A., ApSimon, J. W., and Miller, J. D. (1996) NMR structural studies of fumonisin B1 and related compounds from Fusarium moniliforme, Adv. Exp. Med. Biol. 392, 75-91.

11. Proctor, R. H., Brown, D. W., Plattner, R. D., and Desjardins, A. E. (2003) Co-expression of 15 contiguous genes delineates a fumonisin biosynthetic gene cluster in Gibberella moniliformis, Fungal Genet. Biol. 38, 237-249.

12. Butchko, R. A., Plattner, R. D., and Proctor, R. H. (2003) Esterification of tricarballylic acid side chains to the fumonisin backbone requires the activity of four FUM genes, presented at the Aflatoxin/Fumonisin Elimination and Fungal Genomics Workshop, Savannah, GA.

13. Marahiel, M. A., Stachelhaus, T., and Mootz, H. D. (1997) Modular peptide synthetases involved in nonribosomal peptide synthesis, Chem. Rev. 97, 2651-2674.

14. Reimmann, C., Patel, H. M., Serino, L., Barone, M., Walsh, C. T., and Haas, D. (2001) Essential PchG-dependent reduction in 
pyochelin biosynthesis of Pseudomonas aeruginosa, J. Bacteriol. 183, 813-820.

15. Patel, H. M., and Walsh, C. T. (2001) In vitro reconstitution of the Pseudomonas aeruginosa nonribosomal peptide synthesis of pyochelin: characterization of backbone tailoring thiazoline reductase and $\mathrm{N}$-methyltransferase activities, Biochemistry 40 , 9023-9031.

16. Walsh, C. T., Gehring, A. M., Weinreb, P. H., Quadri, L. E., and Flugel, R. S. (1997) Post-translational modification of polyketide and nonribosomal peptide synthases, Curr. Opin. Chem. Biol. 1, $309-315$

17. Sambrook, J., Fritsch, E. F., and Maniatis, T. (1989) Molecular Cloning: A Laboratory Manual, 2nd ed., Cold Spring Harbor Laboratory Press, New York.

18. Desjardins, A. E., Plattner, R. D., and Proctor, R. H. (1996) Genetic and biochemical aspects of fumonisin production, Adv. Exp. Med. Biol. 392, 165-173.

19. Turgeon, B. G., Garber, R. C., and Yoder, O. C. (1987) Development of a fungal transformation system based on selection of sequences with promoter activity, Mol. Cell. Biol. 7, 32973305.

20. Yu, F., Zhu, X., and Du, L. (2005) Developing a genetic system for functional manipulations of $F U M 1$, a polyketide synthase gene for the biosynthesis of fumonisins in Fusarium verticillioides, FEMS Microbiol. Lett. 248, 257-264.

21. Hartl, M., and Humpf, H. U. (1999) Simultaneous determination of fumonisin $\mathrm{B}(1)$ and hydrolyzed fumonisin $\mathrm{B}(1)$ in corn products by liquid chromatography/electrospray ionization mass spectrometry, J. Agric. Food Chem. 47, 5078-5083.

22. Bojja, R. S., Cerny, R. L., Proctor, R. H., and Du, L. (2004) Determining the biosynthetic sequence in the early steps of the fumonisin pathway by use of three gene-disruption mutants of Fusarium verticillioides, J. Agric. Food Chem. 52, 2855-2860.

23. Kurobane, I., Zaita, N., and Fukuda, A. (1986) New metabolites of Fusarium martii related to dihydrofusarubin, J. Antibiot. 39, 205-214.

24. Brown, D. W., Cheung, F., Proctor, R. H., Butchko, R. A., Zheng, L., Lee, Y., Utterback, T., Smith, S., Feldblyum, T., Glenn, A.
E., Plattner, R. D., Kendra, D. F., Town, C. D., and Whitelaw, C. A. (2005) Comparative analysis of 87,000 expressed sequence tags from the fumonisin-producing fungus Fusarium verticillioides, Fungal Genet. Biol. 42, 848-861.

25. Rheeder, J. P., Marasas, W. F., and Vismer, H. F. (2002) Production of fumonisin analogs by Fusarium species, Appl. Environ. Microbiol. 68, 2101-2105.

26. Weber, G., Schorgendorfer, K., Schneider-Scherzer, E., and Leitner, E. (1994) The peptide synthetase catalyzing cyclosporine production in Tolypocladium niveum is encoded by a giant 45.8kilobase open reading frame, Curr. Genet. 26, 120-125.

27. Butchko, R. A., Plattner, R. D., and Proctor, R. H. (2003) FUM9 is required for $\mathrm{C}-5$ hydroxylation of fumonisins and complements the meitotically defined Fum3 locus in Gibberella moniliformis, Appl. Environ. Microbiol. 69, 6935-6937.

28. Butchko, R. A., Plattner, R. D., and Proctor, R. H. (2003) FUM13 encodes a short chain dehydrogenase/reductase required for C-3 carbonyl reduction during fumonisin biosynthesis in Gibberella moniliformis, J. Agric. Food. Chem. 51, 3000-3006.

29. Ding, Y., Bojja, R. S., and Du, L. (2004) Fum3p, a 2-ketoglutaratedependent dioxygenase required for C-5 hydroxylation of fumonisins in Fusarium verticillioides, Appl. Environ. Microbiol. 70, 1931-1934.

30. Yi, H., Bojja, R. S., Fu, J., and Du, L. (2005). Direct evidence for the function of FUM13 in 3-ketoreduction of mycotoxin fumonisins in Fusarium verticillioides, J. Agric. Food Chem. 53, 54565460.

31. Ehmann, D. E., Trauger, J. W., Stachelhaus, T., and Walsh, C. T. (2000) Aminoacyl-SNACs as small-molecule substrates for the condensation domains of nonribosomal peptide synthetases, Chem. Biol. 7, 765-772.

32. Cane, D. E., Kudo, F., Kinoshita, K., and Khosla, C. (2002) Precursor-directed biosynthesis: biochemical basis of the remarkable selectivity of the erythromycin polyketide synthase toward unsaturated triketides, Chem. Biol. 9, 131-142.

BI052085S 